\title{
Improvements to the two-phase sandwich method for calculating the melting points of pure metals
}

\author{
K. Rybacki*, S. Winczewski, V. Pleechystyy, J. Rybicki \\ Gdańsk University of Technology \\ Faculty of Applied Physics and Mathematics \\ Narutowicza 11/12, Gdańsk, Poland \\ *E-mail: kamil.rybacki@pg.edu.pl
}

Received: 02 June 2019; revised: 23 June 2019; accepted: 25 June 2019; published online: 30 June 2019

\begin{abstract}
The thermophysical properties of metal alloys are often investigated via molecular dynamics (MD) simulations. An exact and reliable estimation of the thermophysical parameters from the MD data requires a properly and carefully elaborated methodology. In this paper, an improved two-phase sandwich method for the determination of the metal melting temperature is proposed, based on the solid-liquid equilibrium theory. The new method was successfully implemented using the LAMMPS software and the $\mathrm{C}++11$ Standard Libraries and then applied to aluminum and copper systems. The results show that the proposed procedure allows more precise calculations of the melting temperature than the widely used onephase boundary methods.
\end{abstract}

Key words: molecular dynamics, melting temperature, phase equilibrium

\section{INTRODUCTION}

Investigations of the thermophysical properties of metals and alloys play an important role for understanding the many phenomena that occurr during metallurgical processes. Obtaining such knowledge may lead to improvements in the preparation of high-performance materials. These materials are widely used, for example in aviation [1], maritime [2] and automotive [3] industries. One of the most investigated topics is the solid-liquid coexistence, which is of great importance for metal alloy casting. One of the parameters describing this state is the equilibrium melting temperature $T_{\text {melt }}$.

The main purpose of calculating the equilibrium melting temperature is to determine whether the liquid alloy is in its normal or undercooled state within the specified range of temperatures. By determining the value of $T_{\text {melt }}$ for varying compositions of a given alloy it is also possible to construct the upper part of its phase diagram which represents the melting process. Due to the physical contact between the melt and the faces of the mold it is often difficult to correctly measure the melting temperatures in the vicinity of the equilibrium melting point. This is caused by a rapid nucleation at the liquid-container boundary which may lead to an underestimation of the melting temperature.

Therefore, calculation methods have developed rapidly in recent years. These methods can be used to predict the value of a $T_{\text {melt }}$ for various metals. These include molecular dynamics simulations that are widely used to investigate the coexistence of liquid and solid phases $[4,5]$ and the thermophysical properties of a wide range of materials in general [6]. Notable applications include transition metals [7, 8], selected rare-earth elements [9] and binary alloys such as $\mathrm{Al}-\mathrm{Cu}[10,11]$ or $\mathrm{Ni}-\mathrm{Zr}$ [12].

From the thermodynamic point of view, the equilibrium melting temperature can be defined as a temperature at which the free enthalpy $G\left(p, T=T_{\text {melt }}\right)$ of the liquid and the solid is identical. The calculations of $T_{\text {melt }}$ by simply heating a periodic fragment of bulk crystal and observing the changes in the thermodynamic functions of state (e.g. the internal energy of the system) to find points of their dis- 
continuous changes lead to overestimated values. This occurs due to the influence of the interfacial energy on the energetics of real, physical systems. Thus, in case of melting temperature calculations it is important to properly prepare the boundary between liquid and solid phases. Considering this requirement, there are currently three methods used for predicting the equilibrium melting temperature which are based on molecular dynamics simulations.

The first and most straightforward method is to carry out solid phase simulations at increasing temperatures. The melting point can be then approximated by the temperature at which the internal crystal lattice structure breaks down. An alternative way to carry out calculations via this method is to cool down a system in a liquid state by subsequently lowering the temperature at which it is equilibrated. Due to the hysteresis effect, the values of $T_{\text {melt }}$ obtained by heating or cooling the chosen simulation system are significantly different from the equilibrium melting temperature. These effects are known as overheating and undercooling, respectively. Basing on the homogeneous nucleation theory it is possible to relate these values to an approximated value of $T_{\text {melt }}[13,14]$. However, this method has been shown to render less precise results than methods based on the two-phase approach [15].

The second method is the moving interface method. Initially, a system is constructed near an estimated value of $T_{\text {melt }}$. It consists of a solid-liquid interface made from solid and liquid phase layers aligned along a chosen coordination axis. Then, the velocity at which the liquidsolid interface moves $v_{\text {int }}$ is calculated. Next, the temperature is changed by the interval $\Delta T$ and the interface moving velocity is measured under new simulation conditions. By investigating a sufficiently wide range of temperatures in the vicinity of $T_{\text {melt }}$, it is possible to plot an interface moving velocity-temperature curve. The extrapolation of this relationship to the point where $v_{\text {int }}=0$ can be treated as an approximation of the melting point. One of the main difficulties of this method is the definition of the solid-phase boundary itself. A clear rule has to be established when a particle becomes a part of the liquid phase during simulations. This can be done using structural analysis methods [16] such as the Common Neighbor Analysis [17] or the Bondorientational order method $[18,19]$. However, if this condition is defined inappropriately, i.e. without taking into account significant thermal excitations of the crystal lattice in the vicinity of the melting point, it may lead to incorrect values of $T_{\text {melt }}$.

The third method is the NVE ensemble method. For its purpose a system similar to the moving interface method can be used [20]. Alternatively, a system consisting of a liquid phase layer located between two solid phase layers can be constructed [21]. This method focuses on the fact that the system consumes latent heat from its surroundings during the solid phase melting. After that, a part of the system's kinetic energy is transferred into its potential energy.
Thus, the overall temperature of the system is lowered. In case when the system is equilibrated in a microcanonical (NVE) ensemble at temperature $T>T_{\text {melt }}$, it evolves towards a lower temperature. Similarly, when the simulation is performed at $T<T_{\text {melt }}$ an increase in temperature towards the melting point is observed. The melting temperature is approximated by the final temperatures at which the system achieves the equilibrium state. It is crucial to ensure that the initial temperature is chosen as close as possible to the equilibrium melting temperature. When done correctly, the NVE method can determine the value of $T_{\text {melt }}$ in a single simulation. This cannot be performed using the moving interface method. However, the two methods can be used to complement each other. First, the melting point can be estimated by a moving interface method. Then, $T_{\text {melt }}$ can be calculated precisely via the NVE method.

Finally, the most precise method used in evaluation of the melting point is the so-called sandwich method $[22,23]$. A detailed description of its theoretical background and problems which arise during its application will be presented in subsequent sections of this article. The main goal of this paper is to establish a repeatable and precise implementation of this method, by addressing the aforementioned challenges. Much attention has been paid to create a computational scheme which can be performed using the readily available scientific software and is characterized by lower computational cost compared to the previously outlined methods.

The paper is organized as follows. In Section II. the basic principles and theoretical background of the sandwich method are recalled. Moreover, the main challenges of this method are addressed and solved in order to ensure precise calculations of the melting temperature and construction of a solid-liquid phase boundary. In Section III. a description of the sandwich method implementation is shown to the reader, using the open-source LAMMPS software [24] and a $\mathrm{C}++$ program used to create a computational system i.e. sandwiches. In Section IV. an example of implementation of the sandwich method is shown for several, available interatomic potentials. As a result, the values of $T_{\text {melt }}$ are calculated and presented for pure aluminum and copper. In Section V. a short discussion is presented in which the authors evaluate whether the proposed implementation of the sandwich method has met the previously posed criteria. Additionally, plans and suggestions of future development of this method are also briefly discussed.

\section{METHODOLOGY}

\section{1. Basic principles}

The sandwich method can be considered as an extension of the hysteresis method. As mentioned earlier, the existence of undercooled and overheated states leads to two different melting points $T^{-}$and $T^{+}$. The true value of $T_{\text {melt }}$ must 
be located in-between those points on the caloric curve of the investigated element, i.e. inside its hysteresis loop, as shown in Fig. 1 by the dimmed portion of the graph.

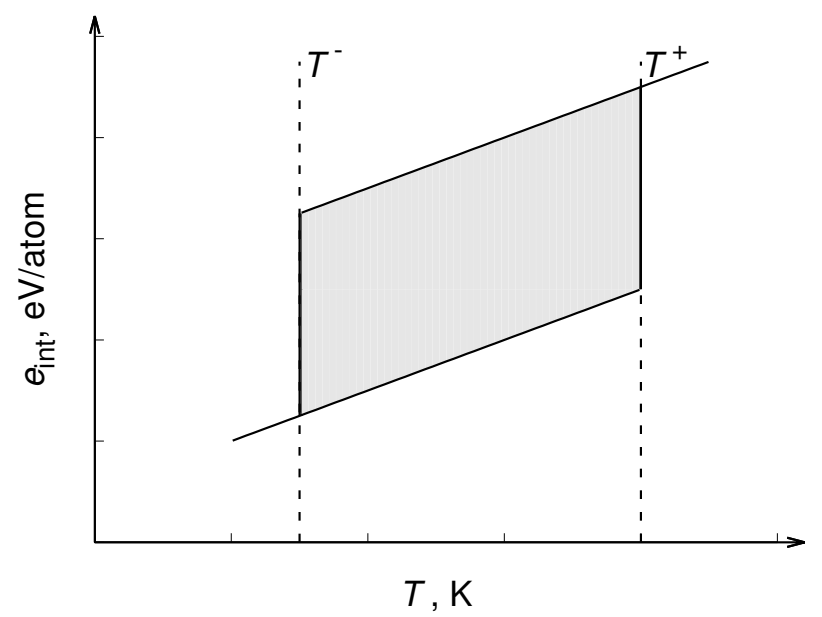

Fig. 1. Example of a caloric curve showing two distinct points of first order phase transitions (freezing and melting)

Therefore, the first step of the sandwich method is to construct a caloric curve where the specific energy (the internal energy per atom) of a one-phase system $e_{\text {int }}$ is plotted against the temperature. This is done for the sake of comparison with future two-phase systems. This can be achieved by heating a bulk solid sample from temperature $T_{\min }$ up to $T_{\max }$ and then cooling the same system back to $T_{\min }$. Simulations are to be carried out in an NpT ensemble. The specific energy of the system $e_{\text {int }}$ is calculated at the temperatures: $T=T_{\min }, T_{\min }+$ $+\Delta T, \ldots, T_{\max }$. The temperature resolution $\Delta T$ and simulation lengths have to be chosen to ensure that the cooling or heating velocity does not lead to the formation of meta-stable phases, e.g. an amorphous glass phase during the cooling of a liquid phase system. Next, the temperatures $T^{-}$and $T^{+}$can be found via the caloric curve analysis. For each sampling temperature $T_{\text {sand }}$ laying between these values, e.g. for $T_{\text {sand }}=T^{-}, T^{-}+\Delta T, \ldots, T^{+}$ a two-phase sandwich is constructed as shown in Fig. 2. Areas (1) and (3) consist of a system in a solid state equilibrated at $T=T_{\text {sand }}$ during the caloric curve creation. Analogically, layer (2) is made of the same element in a liquid state at temperature $T_{\text {sand }}$.

For each set of temperatures $T_{\text {sand }} \in\left(T^{-} ; T^{+}\right)$, a separate simulation is carried out where specific energy is calculated as previously. Initially, these calculations are carried out at time lengths sufficient only for the system to start equilibrating to either the solid or liquid phase. By a superposition of the results obtained for these sandwiches with the caloric curve, a rough estimation of the equilibrium melting point position can be made. Then, for temperatures $T_{\text {sand }}$ in the vicinity of this point it is important to ensure that

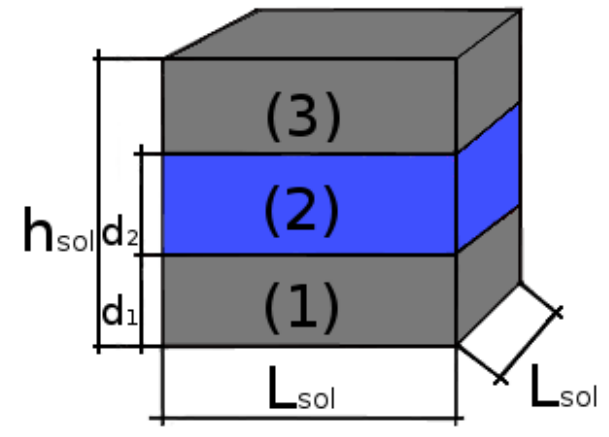

Fig. 2. Schematic showing the type of the simulation systems used in the sandwich method

the system is equilibrated thoroughly, i.e. its energy has converged to a stable value and completely melted or crystallized. Therefore, simulation lengths must be considerably larger than previously. If carried out correctly, an additional discontinuity can be observed at the caloric curve, which corresponds to the equilibrium melting temperature $T_{\text {melt }}$. It is useful to first choose a small subset of temperatures $T_{\text {sand }}$, e.g. which would be equal to $T_{\text {sand }}=800,900, \ldots, 1100 \mathrm{~K}$ for $T^{-}=700 \mathrm{~K}$ and $T^{+}=1200 \mathrm{~K}$ to ensure that the calculations are carried out as efficiently as possible. Then, two sampling points can be identified between which a discontinuity of $e_{\mathrm{int}}^{\mathrm{sand}}$ is observed. These points would act as limits for a subsequent subset of temperatures $T_{\text {sand }}$. This procedure is carried out until a set of temperatures $T_{\text {sand }}$ equidistant from each other by $\Delta T$ is reached. For this last set of temperatures, the sampling stage of simulations is lengthened for a precise estimation of $T_{\text {melt }}$.

\section{2. Preparation of simulation systems}

One of the major requirements of the sandwich method is a reliable construction of the solid-liquid phase interface. Initially, the systems obtained during the equilibration of the liquid and solid system are in the form of orthogonal boxes with the dimensions of $L_{\text {liq }} \times L_{\text {liq }} \times h_{\text {liq }}$ and $L_{\text {sol }} \times L_{\text {sol }} \times h_{\text {sol }}$, respectively. The system used during simulations in the sandwich method can be described as an orthogonal box $h_{\text {sol }}$ in height and with the length of its edges residing in the XY plane being equal to $L_{\text {sol }}$. The system can be divided into three layers to ensure that the concentrations of liquid and solid phases are roughly equal to each other. The thickness of the first and third layers is equal to the quarter of the solid phase simulation box height $h_{\mathrm{sol}}$ : $d_{\text {sol }}=h_{\mathrm{sol}} / 4$. These layers consist of atoms in the solid phase of a given element. A liquid phase layer is inserted between them, the thickness of which is equal to: $d_{l i q}=h_{\text {sol }} / 2$ However, such division is strictly geometric and it does not take into account the thermal excitations of crystal lattices situated in the outermost layers. When applied, it creates 
solid surfaces near the solid-liquid boundary with a high concentration of point defects. Therefore, an algorithm which groups solid phase atoms in subsequent crystal planes along the simulation box height is needed. This can be done by calculating an interatomic distance vector $\mathbf{r}_{i j}^{\text {sol }}$ for each unique pair of solid phase atoms and finding an acute angle $\theta_{i j}^{\text {hkl }}$ between such vector and a crystal plane with Miller indices (hkl). The following equation can be used to calculate this angle:

$$
\theta_{i j}^{\mathrm{hkl}}=\arcsin \left(\frac{\mathbf{r}_{i j}^{\mathrm{sol}}}{\left|\mathbf{r}_{i j}^{\mathrm{sol}}\right|} \cdot \mathbf{n}_{\mathrm{hkl}}\right),
$$

where $\mathbf{n}_{\mathrm{hkl}}$ is a normal to the plane with Miller indices (hkl). A maximum value of $\theta_{i j}^{\text {hkl }}$ can be measured directly using, for example, visualization software such as OVITO [25] by inspecting individual particles in a chosen crystal plane.

One of the problems of this approach is the computational complexity of choosing unique pairs of two solid atoms in a system consisting of $N_{\text {sol }}$ particles. If we use the straightforward method of randomly selecting two atoms in the system, we obtain a complexity proportional to the factor of $N_{\mathrm{sol}}^{2}$. For large systems, it is more efficient to use the Verlet list method. Using this method, the neighbourhood of each particle in the system can be described using two data structures, the number of its closest neighbours and a list containing indices of each of them. During the first step of the Verlet list creation, the distance between two randomly selected atoms $\left|\mathbf{r}_{i j}^{\text {sol }}\right|$ is calculated. If $\left|\mathbf{r}_{i j}^{\text {sol }}\right|$ is smaller than the predefined first coordination sphere radius $r_{\text {sol }}^{\mathrm{I}}$, they are considered closest neighbours. It is important to use the values of $r_{\mathrm{sol}}^{\mathrm{I}}$ which correspond to the structure of an overheated solid at temperature $T^{+}$for which the sandwich is to be constructed. This allows the computational complexity of subsequent interatomic distance calculations to be significantly reduced to the order of $O\left(N_{\mathrm{sol}}^{3 / 5}\right)$. After applying the equation (1) to the closest neighbourhood of a given atom, it is possible to establish which atoms lie at the same crystal planes (hkl). This allows dividing the solid phase into complete crystal planes without introducing previously mentioned additional point defects. A bulk solid phase simulation box is constructed in this way and the periodic boundary conditions are applicable to all three dimensions in this case.

Next, the liquid phase layer must be inserted in the central section of the sandwich. One of the major obstacles in this step are the differences between simulation box sizes of one-phase solid and liquid systems. Due to the equilibration in an NpT ensemble during the caloric curve creation, the volumes of these systems differ in a way that limits the possibility of preserving the periodic boundaries of a liquid phase system by simply dividing it along its height and inserting a part of it inside the sandwich directly. One possibility is to search for areas in the liquid phase called "windows" here, which still preserve its density $\rho_{\text {liq }}$ and their dimensions match the previously defined dimensions of the middle sandwich layer. To measure the density of a single window, it is important to properly define the part of the sandwich volume which will be later used to calculate this property. This definition has to be consistent throughout the whole process of creating a sandwich.

After cutting out a window from the liquid phase system for which $\rho \approx \rho_{\text {liq }}$, it is inserted in the middle layer position inside the sandwich. Then, the bond vectors $\mathbf{r}_{i j}^{\mathrm{ss}}$ between the solid-phase and liquid-phase atoms near the interphase boundary are calculated:

$$
\mathbf{r}_{i j}^{\mathrm{ls}}=\mathbf{r}_{j}^{\mathrm{sol}}-\mathbf{r}_{i}^{\mathrm{liq}}
$$

where $\mathbf{r}_{j}^{\text {sol }}$ is the position vector of one of the solid particles and $\mathbf{r}_{i}^{\text {liq }}$ is the position vector of the liquid particle. Liquid particles which violate the following condition: $r_{i j}^{\mathrm{ls}}=\left|\mathbf{r}_{i j}^{\mathrm{ls}}\right|<r_{\text {cut }}^{\mathrm{sol}}$ are promptly erased from the system. A similar procedure is followed at the boundaries of the sandwich along the $\mathrm{X}$ and $\mathrm{Y}$ axes, where the periodic boundary conditions must apply. Due to the straightforward way in which liquid particles are inserted into the system, the interatomic distances $r_{i j}^{\text {liq }}=\left|\mathbf{r}_{i j}^{\text {liq }}\right|$ must be checked for the liquid atoms residing near those boundaries. An analogical situation occurs if for any pair of liquid atoms $r_{i j}^{\text {liq }}<r_{\text {cut }}^{\text {liq }}$, the particle which originally resides inside the simulation box is removed from the system. Here, we note that in this case the cutoff radii $r_{\text {cut }}^{\text {sol }}$ and $r_{\text {cut }}^{\text {liq }}$ are defined as the smallest possible interatomic distance permitted in the solid and liquid phase systems. Their values can be read directly from the radial distribution function of a given phase as the point of beginning of the first coordination peak. Particles can be removed either all at once, i.e. deleting all the incorrectly placed atoms, or iteratively - one particle at a time. The latter option is more preferable since after erasing one atom the violation of interatomic distances by its neighbours can be eliminated in the process as well. This ensures that only a minimum number of particles is erased from the liquid window and its density still closely matches $\rho_{\text {liq }}$. The total number of atoms inside the sandwich $N_{\text {sand }}=N_{\text {sol }}+N_{\text {liq }}$ has to be recorded in order to calculate its specific internal energy $e_{\text {int }}^{\text {sand }}$ in the future.

When the liquid phase layer has been inserted and the iterative repair algorithm has been applied, the system is ready to be equilibrated at temperature $T_{\text {sand }}$, corresponding to the equilibration conditions of its constituent phases. Similarly to the caloric curve simulations an NpT ensemble is to be chosen, using the same value of external pressure $p$ as for the liquid and solid phases separately. To account for the initial shock present in the system, the sandwich specific internal energy $e_{\text {int }}^{\text {sand }}$ can be calculated as the time average over the last $n_{\text {samp }}$ timesteps leading up to the end of a simulation run. 


\section{IMPLEMENTATION}

\section{1. Hysteresis loop calculations}

Simulations of heating and cooling of a one-phase system were carried out using the LAMMPS [24] software. The initial simulation system was prepared in the form of an orthogonal box containing $20 \times 20 \times 20$ elementary cells. Each of these cells was oriented along the z-axis in the [001] crystallographic direction. The periodic boundary conditions were imposed along all dimensions. This input geometry was used only as an initial condition for simulations in $T=T_{\min }$. For each of the subsequent calculations, the state of the system was recorded at the end of the previous simulation, i.e. it was used for $T^{\prime}=T-\Delta T$.

Each system was simulated in an isobaric-isothermal ensemble. For temperature $T=T_{\min }$, the initial velocities of particles were generated via the Maxwell-Boltzmann distribution. The values of the initial and final temperatures were set at $T_{\min }=T_{\text {melt }}^{\prime}-600 \mathrm{~K}$ and $T_{\max }=T_{\text {melt }}^{\prime}+600 \mathrm{~K}$, where $T_{\text {melt }}^{\prime}$ was an experimental value of the equilibrium melting temperature for a given element. The temperature resolution was set to $\Delta T=5 \mathrm{~K}$. The length of the simulation runs for subsequent temperatures $T$ was set to 150000 timesteps, where one timestep $\delta_{t}=1 \mathrm{fs}$. The first 50000 timesteps were treated as an equilibration run and the rest of the simulation run was devoted to the sampling of system thermodynamic parameters, i.e. $e_{\text {int }}, T$ and pressure tensor elements $p_{i j}$. During the calculations the microstate of the system was registered at reach timestep for future construction of two-phase sandwiches.

Having known the total number of particles in the system $N$ at each temperature, the specific internal energy $e_{\text {int }}$ was calculated using the following formula:

$$
e_{\mathrm{int}}=\frac{E_{\mathrm{int}}}{N}
$$

The temperatures $T^{-}$and $T^{+}$were estimated for a given element by analyzing the specific internal energy hysteresis loop $e_{\text {int }}(T)$. In the case when the specific internal energy of a system does not show any discontinuity at a single temperature, but changes continuously towards the opposite part of the hysteresis loop, the values of $T_{\min }$ for freezing and $T_{\max }$ for heating are chosen as the last temperatures before the decline or raise of $e_{\text {int }}$, as shown in Fig. 3a. This is most often observed at the cooling stage of simulations, where for the applied potential model the cooling rate used during equilibration of the system leads to the glass transition of the undercooled liquid. If this effect leads in the end to the formation of the amorphous glass phase instead of the equilibrium crystal phase, the lengths of the equilibration and sampling periods are doubled, so the cooling rate is lowered. Another factor taken into account is the presence of other meta-stable crystal phases occurring in the case of potential models such as the ADP model of Mishin et. al. [26].
This is evident by a slight difference of the specific internal energy of solid phase systems registered before and after heating, as shown in Fig. 3b. In this case, the temperature $T^{-}$is chosen as previously for the systems, in which $e_{\text {int }}(T)$ does not show a discontinuity in the vicinity of the freezing point.

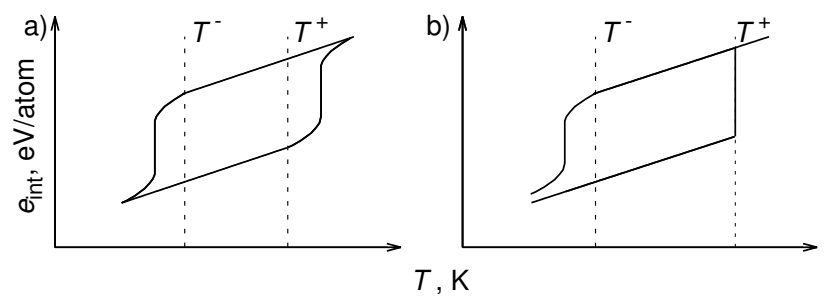

Fig. 3. Two possible computational artifacts prone to appearing during hysteresis loop simulations

In order to generate double interphase boundary systems used for calculations of the equilibrium melting temperature $T_{\text {melt }}$, an application was created using the $\mathrm{C}^{++11}$ Standard Library. The basic operating principle of this software is as follows.

\section{2. Constructing two-phase systems - sandwiches III. 2. 1. Initial conditions}

First, the program reads two LAMMPS trajectory files one for the solid phase system and one for the liquid one, both equilibrated at the same temperature $T$. The parser implemented for reading .lammpstrj files reads only a final microstate of the system, i.e. the last snapshot of the sampling period. The dimensions $h$ and $L$ of the simulation box and the total number of particles $N$ present in it are the first parameters parsed from the trajectory file. Each dimension is represented in that file as a minimum and maximum value of a given coordinate, e.g. we obtain $x_{\mathrm{lo}}$ and $x_{\mathrm{hi}}$ along the $\mathrm{X}$-axis. Thus, length $L$ can be computed later as a difference of these parameters. At this stage, the reference atomic densities for solid and liquid phases are calculated as a ratio of the number of atoms present in the simulation box $N$ and its volume: $V=h L^{2}$. Next, the Cartesian coordinates and velocities recorded at the last timestep are read and each atom is assigned an atom type corresponding to the type of phase to which it belongs. For example, when a solid phase trajectory file is read, each atom is assigned a particle type equal to 1 . This is done to differentiate particles during the construction and future simulations of sandwiches. Due to the fact that LAMMPS only wraps atom coordinates in periodic boundary boxes on timesteps when Verlet lists are created, these coordinates are then wrapped back into the simulation box if necessary. This is done simply by checking if a given coordinate lies within the simulation box boundaries read at the beginning of the trajectory file. The data obtained during the parsing of an input file is stored inside a structure called phase, which contains information about 
the simulation box dimensions, the volume of the system, the number and type (solid or liquid) of atoms. Information about velocity and position vectors is stored within a pointerlist of structures of the atom type. This structure contains the position of a given particle expressed in absolute and partial coordinates, its atom type, components of the velocity vector and a Boolean variable which states if the particle was deleted from the system at any stage of the program. Additionally, solid phase atoms are later assigned two additional parameters. The first of these is an integer ranging from 1 up to $n_{\text {planes }}$, which signifies into which crystal plane the atom has been grouped. The second one is a Boolean variable which tells the program if the atom has been assigned to any of those crystal planes. The parameter $n_{\text {planes }}$, which is the number of crystal planes present in the solid phase, is obtained from their identification performed after reading the input files.

\section{2. 2. Identification of crystal planes in solid phase}

After reading the input files, the program then proceeds to analyze the solid phase data stored inside one of the phase structures created previously. First, a neighbour list is created based on the first coordination sphere radius for the solid phase $r_{\text {sol }}^{\mathrm{I}}$. Its value is obtained from the radial distribution function as the radius located between the first and second coordination peaks. This is done to ensure that the whole first coordination sphere is considered, with thermal excitations of the crystal lattice taken into account. Additionally, periodic boundary conditions are enabled in all the three directions. As a result, a tree-like data structure is created, in which each atom is assigned the indices of its closest neighbours. Next, a breadth-first search (BFS) algorithm is performed. As the first step, one of the atoms is chosen randomly in the system as a "search key". If it has been assigned to any planes previously, it is promptly skipped. Then, the equation (1) is applied for each of its neighbours. The neighbours which satisfy these criteria are assigned to the same crystal plane as the "search key" atom and then taken as starting points of the next iteration of the algorithm. In other words, after considering the neighbours of the first "search key" atom, each of those neighbours serves as a root of a subtree for which the same procedure is applied. This happens until the queue of next subtree roots is empty, i.e. all atoms laying in the same crystal plane have been found. Then, the system is searched for the next "search key" atom and another crystal plane is identified. At each step when a new "search key" is found, a variable signifying the number of crystal planes $n_{\text {planes }}$ is incremented. The BFS algorithm is stopped when there are no more atoms which have not been assigned to any crystal plane. Fig. 4 shows the crystal planes identified by this procedure.

After the end of the identification process, an average height $\left\langle z_{i}\right\rangle$, where $i=1,2, \ldots, n_{\text {planes }}$, is calculated for each crystal plane. Using those values it is possible to geometrically divide the sandwich simulation box into three distinct layers, whilst eliminating the occurrence of defected solid phase planes. Moreover, an interplanar distance $d_{\mathrm{hkl}}$ can be obtained by dividing the simulation box height $h_{\mathrm{sol}}$ by the number of the identified crystal planes: $d_{\mathrm{hkl}}=$ $=h_{\text {sol }} / n_{\text {planes }}$.

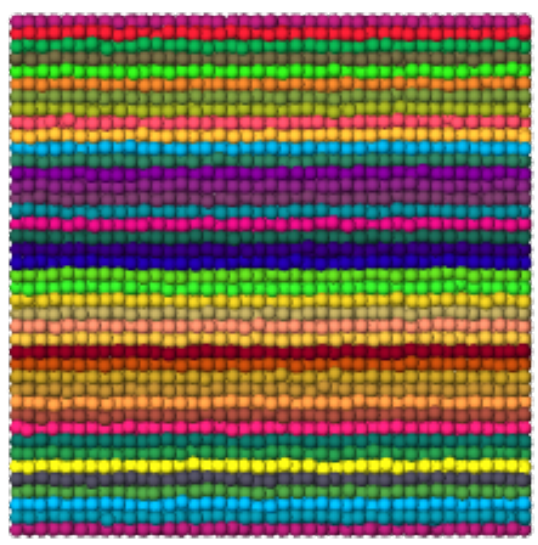

Fig. 4. Visualization of atoms grouped into subsequent crystal planes along $\mathrm{Oz}$ axis in OVITO [25]

Thus, it is possible to properly define the volume occupied by a single solid phase layer. For example, the thickness $d_{1}$ of the bottom layer (marked as layer 1 in Fig. 2) can be expressed as:

$$
d_{1}=\langle z\rangle_{\max }+\frac{d_{\mathrm{hkl}}}{2},
$$

where $\langle z\rangle_{\max }$ is the average height of the uppermost crystal plane of that layer. A symmetrical expression can be written for the uppermost layer:

$$
d_{3}=\left(h_{\mathrm{sol}}-\langle z\rangle_{\mathrm{min}}\right)+\frac{d_{\mathrm{hkl}}}{2},
$$

where $\langle z\rangle_{\min }$ is the average height of the lowermost crystal plane included in that layer. Due to the way in which the simulation box has been divided, the values for $\langle z\rangle_{\min }$ and $\langle z\rangle_{\max }$ are roughly $1 / 4 h_{\text {sol }}$ and $3 / 4 h_{\text {sol. }}$. Thus, the liquid phase layer thickness must be approximately equal to: $d_{2} \approx 1 / 2 h_{\mathrm{sol}}-d_{\mathrm{hkl}}$. The solid phase atoms for which $\left\langle z_{i}\right\rangle$ resides between the values $\langle z\rangle_{\min }$ and $\langle z\rangle_{\max }$ are flagged as removed for further construction of sandwich systems.

\section{2. 3. Sampling of liquid phase microstate}

After inserting solid crystal planes into the sandwich system with thicknesses $d_{1}$ and $d_{3}$, the program proceeds to sample the liquid phase microstate using an orthogonal box of dimensions $d_{2} \times L_{\mathrm{sol}} \times L_{\mathrm{sol}}$. Here, a slight modification to the value of $d_{2}$ parameter is made. Using $d_{2} \approx$ $\approx 1 / 2 h_{\mathrm{sol}}-d_{\mathrm{hkl}}$, there are positions of liquid atoms which 
would be normally permitted in the interphase boundary, but are not present there due to the insufficient thickness of the liquid phase layer. Furthermore, this would also create planar voids in-between the solid and liquid phase layers. Thus, by choosing $d_{2} \approx 1 / 2 h_{\text {sol }}$, we can eliminate both these concerns by following the procedure presented in Section II. 2 .

During the sampling process, $M$ random points are chosen inside the liquid phase simulation box. These points act as centers for liquid layer "windows", which will be later inserted into sandwich systems. From now on, we will denote the position vector of one of these centers as $\mathbf{r}_{0}=$ $\left(x_{0}, y_{0}, z_{0}\right)$. The boundaries of one of these windows can be expressed as: $\alpha_{\mathrm{low}}^{w}=\alpha_{0}-0.5 L_{\alpha}$ and $\alpha_{\mathrm{hi}}^{w}=\alpha_{0}+0.5 L_{\alpha}$, where $\alpha \in\{x, y, z\}$ and $L_{\alpha}$ is the dimension of the window along $\alpha$-axis. If any of these parameters exceeds any of the sandwich simulation box boundaries, the periodic boundary conditions are taken into consideration. Next, the spatial coordinates of each particle in liquid phase systems are checked to see which of them reside inside the window boundaries. If an atom is contained within this box, its position vector is expressed in terms relative to the chosen window center $\mathbf{r}_{0}$ as:

$$
\mathbf{r}_{i}^{\prime}=\mathbf{r}_{i}^{\mathrm{liq}}-\mathbf{r}_{0}
$$

Using this description of the atomic positions within the liquid phase layer allows a simplified way of inserting those particles into the sandwich simulation box. This can be done by an inverse transformation where $\mathbf{r}_{0}$ is set to the center point of the whole sandwich:

$$
\begin{aligned}
\mathbf{r}_{0} & =\left(\frac{L_{\mathrm{sol}}}{2}, \frac{L_{\mathrm{sol}}}{2}, \frac{h_{\mathrm{sol}}}{2}\right), \\
\mathbf{r}_{i}^{\text {liq }} & =\mathbf{r}_{i}^{\prime}+\mathbf{r}_{0} .
\end{aligned}
$$

For each liquid phase the window atomic density is calculated as a measure of its quality. It can be defined as a ratio between the number of particles included within its boundaries $N_{\text {in }}$ and its volume $V_{\text {win }}=d_{2} L_{\text {sol }}^{2}: \rho_{\text {win }}=N_{\text {in }} / V_{\text {win }}$. Next, a ratio of $\rho_{\text {win }}$ to $\rho_{\text {liq }}$ is calculated for each window and all of them are sorted based on this parameter in an ascending order. From this population of windows, $M^{\prime}$ best are chosen as candidates for the next step. The parameters $M$ and $M^{\prime}$ are usually chosen, so that: $M^{\prime} / M \approx 0.1(10 \%)$.

\section{2. 4. Testing of sandwiches}

After finding a population of liquid windows suitable for insertion as a middle layer in the sandwich, the program proceeds to check which atoms violate the interatomic distance requirements outlined in Section II. 2. Liquid particles are inserted into the sandwich simulation box using Eq. (7) and Eq. (8). Next, a modified neighbour list is created for each liquid atom in the system. Instead of using the first coordination sphere radii $r_{\text {sol }}^{\mathrm{I}}$ and $r_{\text {liq }}^{\mathrm{I}}$, the cutoff radii $r_{\text {cut }}^{\text {sol }}$ and $r_{\text {cut }}^{\text {liq }}$ are used. Their definition is presented in Section II. 2. The following rules are applied when checking the interatomic distances $\mathbf{r}_{i j}$. When atom $i$ is of the liquid type and atom $j$ is of the solid type, the cutoff radius used is equal to $r_{\text {cut }}^{\text {sol }}$ to account for the special cases presented in the previous subsection. When both atoms are of the liquid type, $r_{\text {cut }}^{\text {liq }}$ is used instead of $r_{\text {cut }}^{\text {sol }}$. Thus, the Verlet list created will contain only those atoms which are too close to each other. This occurs mainly at the simulation box boundaries in the middle sandwich layer and at the liquid-solid phase boundaries. For each of those particles an imperfection factor $\Delta r_{i}$ is assigned which can be calculated as:

$$
\Delta r_{i}=\frac{\sum_{j}^{n_{\text {neigh }}}\left(r_{\text {cut }}-\left|\mathbf{r}_{i j}\right|\right)}{n_{\text {neigh }}},
$$

where $n_{\text {neigh }}$ is the number of neighbours of an $i$-th atom. This data is kept in the Verlet list for each atomic pair alongside information about interatomic distances, etc. In the liquid phase region there is a high possibility that a significant number of atoms will exhibit some kind of imperfection, i.e. small values of $\Delta r_{i}$, due to the way its microstate was sampled. To account for that, a histogram of $\Delta r_{i}$ is created and later analyzed in order to define a maximum value for this parameter $\Delta r_{\max }$ to be permitted in the sandwich system. An example of such graph is shown in Fig. 5.

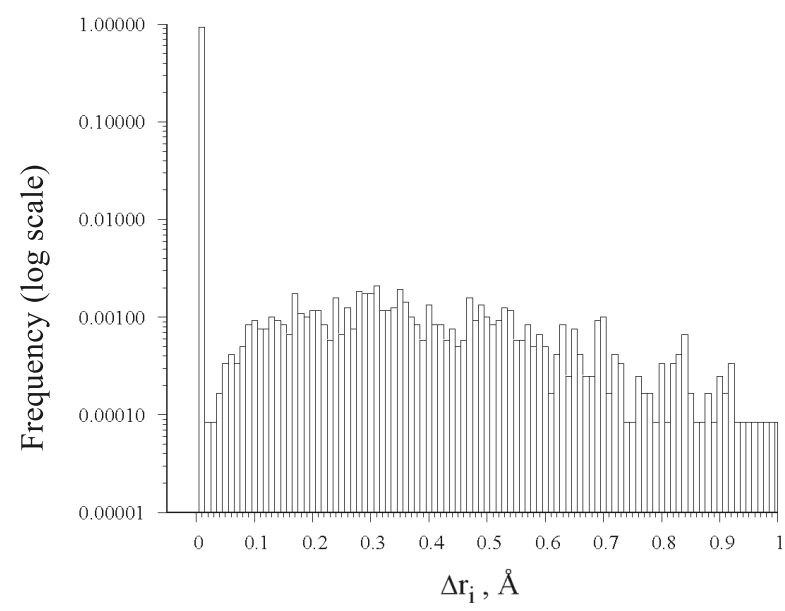

Fig. 5. Histogram of the imperfection parameter made before applying a repairing procedure for a sandwich system. The frequency here is defined as the number of atoms which reside in the given bin due to their values of $\Delta r_{i}$ divided by the total number of liquid particles in the system $N_{\text {liq }}$. Each bin has the width equal to $0.01 \AA$.

The data was averaged over 10 different prepared sandwiches

As seen on the example of a histogram, there is a dominant group of particles for which the imperfection factor is in an acceptable range (around $0.01 \AA$ ). Thus, the upper limit of this histogram bin can be taken as a value for $\Delta r_{\max }$. Any groups of atoms for which $\Delta r_{i}>\Delta r_{\max }$ are classified as defective atoms in the future iterative repair algorithm. 
To ensure that only a minimum number of liquid atoms is removed from the system, an iterative scheme is implemented. As the first step, a maximum value of $\Delta r_{i}$ is found using the previously created Verlet list. This coincides with the rightmost bin in Fig. 5. Then, one of the atoms from this group is removed from the system. This is done by deleting information about this particle contained in its neighbour entries from the Verlet list. Next, the parameter $\Delta r_{i}$ is recalculated for all atoms still present in the neighbour list and a new version of the histogram is created. This is repeated until $\Delta r_{i}$ for all atoms in the system is lower than the previously defined $\Delta r_{\max }$. After that, the final density $\rho_{\text {fin }}$ of the middle sandwich layer is calculated as a ratio of the number of the liquid atoms left in the system $N_{\text {liq }}^{\prime}$ and the volume of the repaired middle layer as $V_{\text {win }}^{\prime}=0.5 h_{\text {sol }}$. Using this approach is more complex than deleting all faulty particles at once; however, the results in sandwiches for which the density $\rho_{\text {fin }}$ is still comparable with the reference density of the liquid phase $\rho_{\text {liq }}$ i.e: $N_{\text {liq }}-N_{\text {liq }} \rightarrow$ min. The effect of that is shown in Fig. 6.

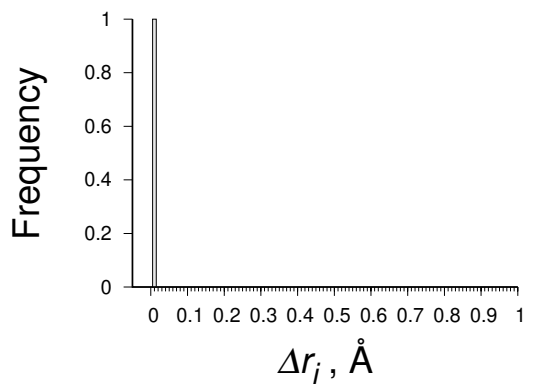

Fig. 6. Histogram of the imperfection parameter after applying a repairing procedure for a sandwich system. Each bin has the width equal to $0.01 \AA$. All atoms in the system reside only in the first bin. This means that the imperfection factor $\Delta r_{i}$ for all particles has been lowered to the maximum value of $\Delta r_{\max }=0.01 \AA$. The data was averaged over 10 different prepared sandwiches

\section{2. 5. Validation of iterative repair algorithm}

To further validate this implementation, a numerical experiment was carried out to determine if there was any privileged way of choosing the initial liquid phase windows for the repair algorithm. In other words, we want to check the existence of a "perfect" window by investigating if there is any correlation between the initial $\rho_{\text {win }}$ and the final $\rho_{\text {fin }}$ window density. In theory, if there is any relationship present between those parameters, there should also be a way to choose only those initial windows with the density $\rho_{\text {win }}$ so that $\frac{\rho_{\text {fin }}}{\rho_{\text {liq }}} \approx 1$. This would mean that an additional condition should be applied to filter out those "perfect" windows (based on their $\rho_{\text {win }}$ ) total population of $M$ randomly chosen samples. For this purpose, the $M=100$ random liquid windows were chosen as a test group. For each of them, the liquid phase atomic density before and after the repairing algorithm was calculated $-\rho_{\text {win }}$ and $\rho_{\text {fin }}$, respectively. The results of this experiment are presented in Fig. 7.

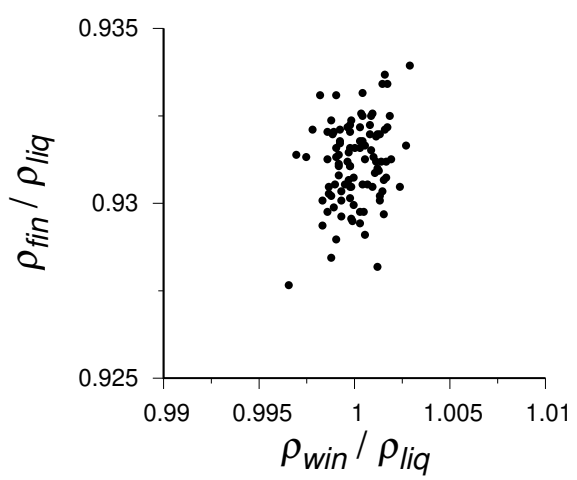

Fig. 7. Densities of chosen liquid windows before and after iterative repair algorithm, related to the reference atomic density of the liquid phase

Based on this data, it was determined that there was no correlation between the initial and final atomic densities of liquid phase windows using the iterative repair algorithm. The way in which their location is chosen within a liquid phase simulation box does not affect the construction of twophase sandwiches directly. Due to the removal of liquid particles at the interphase boundaries, the final atomic densities are almost always lower relative to their initial value by a factor of $\sim 6 \%$. Thus, the iterative repair algorithm does not distinguish any privileged liquid phase windows for which it is possible to obtain $\rho_{\text {fin }} \approx \rho_{\text {liq }}$.

\section{2. 6. Writing LAMMPS geometry (data) files}

After a group of $M^{\prime}$ liquid windows were tested, a similar sorting algorithm was used as in Section III. 2. 3. using their final densities $\rho_{\text {fin }}$ as sorted values. From this group the $M_{\text {best }}$ number of windows is used for the creation of LAMMPS data files containing sandwich systems. Here this parameter is chosen, so that: $M_{\text {best }} / M^{\prime} \approx 0.1$. This means that only $1 \%$ best out of $M$ initial windows are considered as suitable to be used in molecular dynamics simulations. Then, a sandwich is created for each of those samples using Eq. (7) and Eq. (8). Having expressed the positions of all particles in such Cartesian coordinates and the velocity vector components stored from reading of one-phase input files, it is now possible to create input geometry files to be used in the LAMMPS software. Faulty liquid particles which were identified during the repair algorithm were flagged as removed by modifying the Boolean variable stored inside their respective atom data structures. This means that by checking the value of this variable, the program can write data about particles which are still present inside the sandwich. Similarly, this is done for solid particles as well. The total number of atoms $N_{\text {sand }}$ in the system is taken as a sum of $N_{\mathrm{sol}}$ and the number of liquid particles left after the repair 
scheme $N_{\text {liq }}^{\prime}$. The simulation box boundaries are identical to the boundaries of a one-phase solid system.

\section{2. 7. Molecular dynamics of sandwiches}

Each sandwich created by the program is then equilibrated in its respective temperature $T_{\text {sand }}$, i.e. in a temperature in which the solid and liquid phases were sampled. This is done using the LAMMPS software again. The simulations carried out during the rough estimation of $T_{\text {melt }}$ were $n_{\text {samp }}=200000$ timesteps in length. For precise calculations of the equilibrium melting point, this parameter was set to $n_{\text {samp }}=1000000$ timesteps. One timestep was equal to $\delta t=1 \mathrm{fs}$. All the simulations were carried out in the $\mathrm{NpT}$ ensemble for the external pressure equal to $p=0$ bar, i.e. in vacuum.

\section{APPLICATION}

The equilibrium melting temperatures $T_{\text {melt }}$ for aluminum and copper were calculated using the angular dependent potential (ADP) developed by Apostol et. al. [26] and the embedded atom method (EAM) potential of Mishin [27].

The hysteresis loop calculations and the sandwich method simulations were carried out according to the specifications outlined in Sec. III.. The hysteresis loop shown in Figs. 8 to 11 serves as an illustration of the equilibrium melting temperature $T_{\text {melt }}$ estimation in the sandwich method. As written in Section II., the quantity of the sampling points increases as each subset temperature $T_{\text {sand }}$ approaches an estimated value of $T_{\text {melt }}$. In case of shorter lengths of simulations ( $n_{\text {samp }}=200000$ timesteps), the values of $e_{\text {int }}^{\text {sand }}$ can be located between the lower and upper bounds of the hysteresis loop. This effect occurs most prominently in the vicinity of the melting point. To counter this,

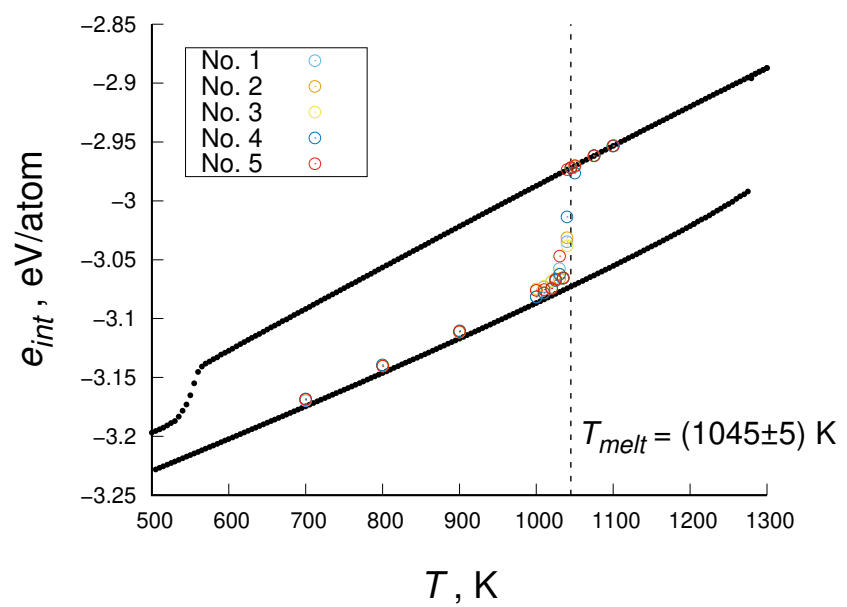

Fig. 8. Sandwich method hysteresis loop calculated for aluminum using the ADP potential

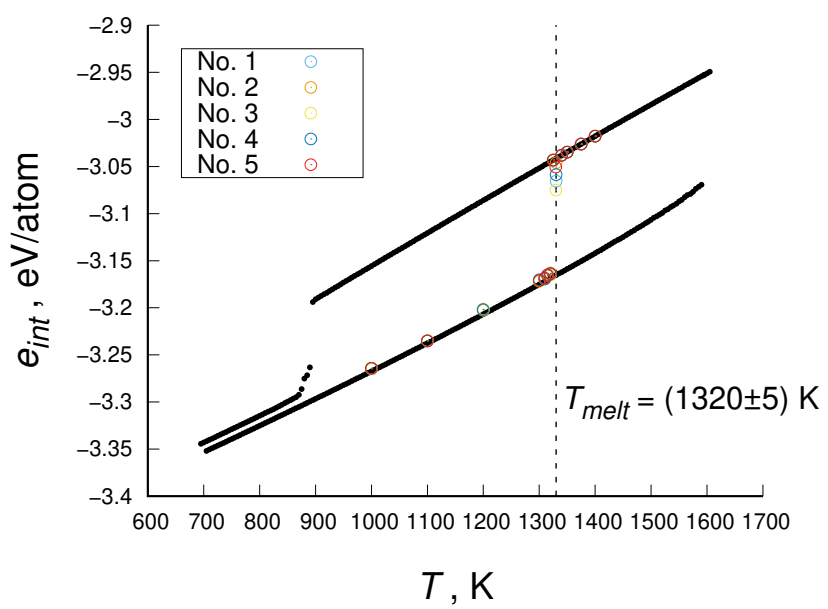

Fig. 9. Sandwich method hysteresis loop calculated for copper using the ADP potential

these systems were simulated once more using a larger number of sampling timesteps as discussed previously.

To eliminate problems which may result from the quality of the created simulation systems, 5 different sandwiches were constructed for each sampling point $T_{\text {sand }}$. These are labeled in different colors in the aforementioned figures.

In the case of the ADP potential, the difference of specific energies between the equilibrium solid and the quenched liquid was observed, although no formation of the amorphous glass phase in the vicinity of the $T^{-}$temperature occurred. A structural analysis via the BOP method was performed, which showed the existence of icosahedral substructures within the cooled liquid systems.

The calculated values of the equilibrium melting temperature $T_{\text {melt }}$ for each element and the potential model used are shown in Tab. 1. These values are compared with the experimental data and the results obtained by other authors by means of alternative methods.

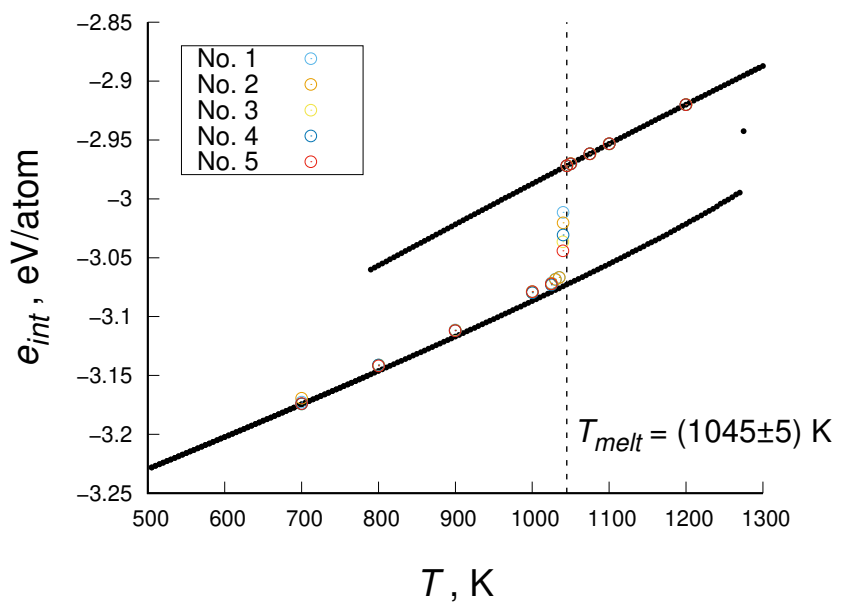

Fig. 10. Sandwich method hysteresis loop calculated for aluminum using the EAM-Mishin potential 


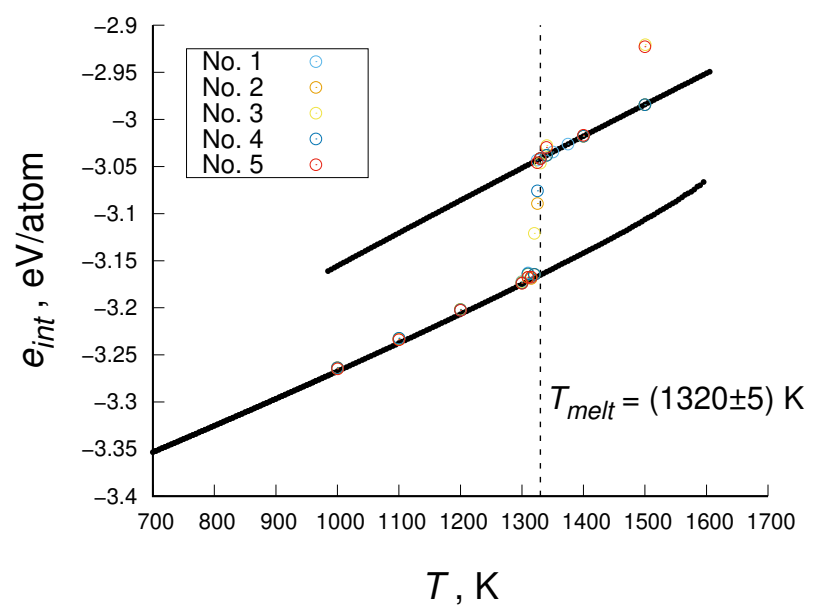

Fig. 11. Sandwich method hysteresis loop calculated for copper using the EAM-Mishin potential

Tab. 1. Equilibrium melting temperatures calculated using the improved sandwich method, compared with experimental and $a b$ intio results. Error of measurements via the sandwich method was set to the smallest resolution of temperatures used during the simulations i.e. $\Delta T$

\begin{tabular}{rrr}
\hline Element & Source & $T_{\text {melt }}, \mathrm{K}$ \\
\hline \multirow{2}{*}{ Al } & ADP & $1045 \pm 5$ \\
& EAM-Mishin & $1050 \pm 5$ \\
& EAM-Mishin [28] & $1044 \pm 34$ \\
& Ab intio [29] & $890 \pm 20$ \\
& Experimental [30] & 933.47 \\
\hline \multirow{2}{*}{ Cu } & ADP & $1330 \pm 5$ \\
& EAM-Mishin & $1320 \pm 5$ \\
& EAM-Mishin [28] & $1333 \pm 50$ \\
& Ab intio [31] & $1251 \pm 15$ \\
& Experimental [32] & $1354 \pm 5$ \\
\hline
\end{tabular}

\section{SUMMARY}

In contrast to alternative methods, the sandwich method imposes strict requirements for the creation of simulation systems and their equilibration. Imposing those constraints upon the algorithm of the simulation system preparation resulted in two-phase systems, where the influence of defects such as the interphase boundary or defected crystal surfaces on the overall energetics of the system was minimized. This can be seen when analyzing the hysteresis loops shown in Fig. 8 and Fig. 9. In both cases all the simulation systems, i.e. sandwiches underwent the phase transition process at exactly the same temperature. This means that they represent a set of initial states equivalent to each other from the macro- scopic point of view, i.e. they represent the same thermodynamic state being sampled at different instants of time. Additionally, the two-phase sandwich method was improved in terms of the measurement error of equilibrium melting temperature. Moreover, this temperature was in this case well defined, based on theory of the first order phase transitions. These factors suggest that $T_{\text {melt }}$ can be calculated precisely with our improved method.

The results obtained by the sandwich method for the EAM-Mishin potential are in agreement with the alternative one boundary method used by the authors of [28]. The discrepancies between those results and ab intio or the experimental data can occur due to the fact that most of the potentials for the $\mathrm{Al}-\mathrm{Cu}$ systems are parameterized with the $\theta$ and $\theta^{\prime}$ phases in mind. This means that those models are best applicable in temperatures significantly lower that the melting point of a given element. This is especially evident in case of aluminum. For the ADP model, we were unable to find any results for the equilibrium melting temperatures of the investigated elements obtained via other onephase methods. Given the fact that this model was parameterized with similar goals as EAM-Mishin, i.e. to predict the existence of metastable $\mathrm{AlCu}_{2}$ phases, calculations were carried out to check if the above hypothesis was correct. By comparing the results obtained for the ADP model with the reference data it can be seen that those discrepancies are independent of the method used for the estimation of melting points. Further investigations are to be carried out using the potentials created specifically for modeling of phase transitions which occur during melting of $\mathrm{Al}-\mathrm{Cu}$ alloys.

\section{References}

[1] Y. Kaushik, A review on use of aluminium alloys in aircraft components, i-manager's Journal on Material Science 3, 33-38 (2015).

[2] S. Ferraris, L.M. Volpone, Aluminium alloys in third millennium shipbuilding: Materials, technologies, perspectives, The Fifth International Forum on Aluminum Ships, 1-11 (2005).

[3] J. Hirsch, Recent development in aluminium for automotive applications, Transactions of Nonferrous Metals Society of China 24, 1995-2002 (2014).

[4] Y. Hiwatari, E. Stoll, T. Schneider, Molecular-dynamics investigation of solid-liquid coexistence, J. Chem. Phys. 68(8), 3401-3404 (1978).

[5] S. Toxvaerd, E. Praestgaard, Molecular dynamics calculation of the liquid structure up to a solid surface, J. Chem. Phys. 67(11), 5291-5295 (1977).

[6] Y.J. Lv, M. Chen, Thermophysical properties of undercooled alloys: An overview of the molecular simulation approaches, International Journal of Molecular Sciences 12(1), 278-316 (2011).

[7] X. Liu, X. Wen, R. Hoffmann, Surface activation of transition metal nanoparticles for heterogeneous catalysis: What we can learn from molecular dynamics, ACS Catalysis 8(4), 3365-3375 (2018). 
[8] C.F. Sanz-Navarro, P.O. Åstrand, D. Chen, M. Rønning, A.C.T. van Duin, W.A. Goddard, Molecular dynamics simulations of metal clusters supported on fishbone carbon nanofibers, J. Phys. Chem. C 114(8), 3522-3530 (2010).

[9] M. Matsumiya, K. Seo, A Molecular Dynamics Simula-

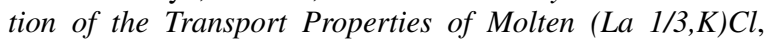
Zeittschrift für Naturforschung A 60, 187-192 (2005).

[10] M.E. Trybula, Structure and transport properties of the liquid Al $80 \mathrm{Cu} 20$ alloy - A molecular dynamics study, Computational Materials Science 122 (2016).

[11] J. Rybicki, J. Dziedzic, S. Winczewski, Structure and properties of liquid Al-Cu alloys: Empirical potentials compared, Computational Materials Science 114, 219-232 (2016).

[12] F.R. Eshelman, J.F. Smith, Single-crystal elastic constants of Al2Cu, Journal of Applied Physics 49(6), 3284-3288 (1978).

[13] S.N. Luo, A. Strachan, D.C. Swift, Nonequilibrium melting and crystallization of a model Lennard-Jones system, J. Chem. Phys. 120, 11640 (2004).

[14] S.N. Luo, T.J. Ahrens, Superheating systematics of crystalline solids, Applied Physics Letters 82(12), 1836-1838 (2003).

[15] W. Zhang, Y. Peng, Z. Liu, Molecular dynamics simulations of the melting curve of NiAl alloy under pressure, AIP Advances 4, 057110 (2014).

[16] A. Stukowski, Structure identification methods for atomistic simulations of crystalline materials, Modelling and Simulation in Materials Science and Engineering 20 (2012).

[17] P.M. Larsen, S. Schmidt, J. Schiøtz, N. Ummen, T. Kraska, Common neighbour analysis for binary atomic systems Recent citations Common neighbour analysis for binary atomic systems, Modelling Simul. Mater. Sci. Eng 15, 319-334 (2007).

[18] P.J. Steinhardt, D.R. Nelson, M. Ronchetti, Icosahedral bond orientational order in supercooled liquids, Phys. Rev. Lett. 47, 1297-1300 (1981).

[19] S. Winczewski, J. Dziedzic, J. Rybicki, A highly-efficient technique for evaluating bond-orientational order parameters, Computer Physics Communications 198, 128-138 (2016).
[20] S. Yoo, X.C. Zeng, J.R. Morris, The melting lines of model silicon calculated from coexisting solid-liquid phases, J. Chem. Phys. 120(123), 1654-1656 (2004).

[21] J.R. Morris, X. Song, The Melting Lines of Model Systems Calculated from Coexistence Simulations, Chemical Physics 116 (2002).

[22] S. Maćkowiak, S. Pieprzyk, A.C. Brańka, D.M. Heyes, A Nosé-Hoover Thermostat Adapted to a Slab Geometry, CMST 23(3), 211-218 (2017).

[23] Y. Zhang, E.J. Maginn, A comparison of methods for melting point calculation using molecular dynamics simulations, J. Chem. Phys. 136 (2012).

[24] S. Plimpton, Fast Parallel Algorithms for Short-range Molecular Dynamics, J. Comput. Phys. 117(1), 1-19 (1995).

[25] A. Stukowski, Visualization and analysis of atomistic simulation data with OVITO - the open visualization tool, Modelling and Simulation in Materials Science and Engineering 18(1) (2010).

[26] F. Apostol, Y. Mishin, Interatomic potential for the Al-Cu system, Phys. Rev. B 83, 054116 (2011).

[27] Y. Mishin, M.J. Mehl, D.A. Papaconstantopoulos, A.F. Voter, J.D. Kress, Structural stability and lattice defects in copper: Ab initio, tight-binding, and embedded-atom calculations, Phys. Rev. B 63 (2001).

[28] X.W. Zhou, D.K. Ward, M.E. Foster, An analytical bondorder potential for the aluminum copper binary system, Journal of Alloys and Compounds 680, 752-767 (2016).

[29] G.A. De Wijs, G. Kresse, M.J. Gillan, First-order phase transitions by first-principles free-energy calculations: The melting of Al, Phys. Rev. Lett. 74, 1823-1826 (1995).

[30] D.R. Lide, CRC Handbook of Chemistry and Physics, American Chemical Society, Boca Raton, 87th ed. (2006).

[31] L.F. Zhu, B. Grabowski, J. Neugebauer, Efficient approach to compute melting properties fully from ab initio with application to $\mathrm{Cu}$, Phys. Rev. B 96 (2017).

[32] H. Brand, D.P. Dobson, L.V. Cadlo, I.G. Wood, Melting curve of copper measured to 16 GPa using a multi-anvil press, High Pressure Research 26(3), 185-191 (2006). 

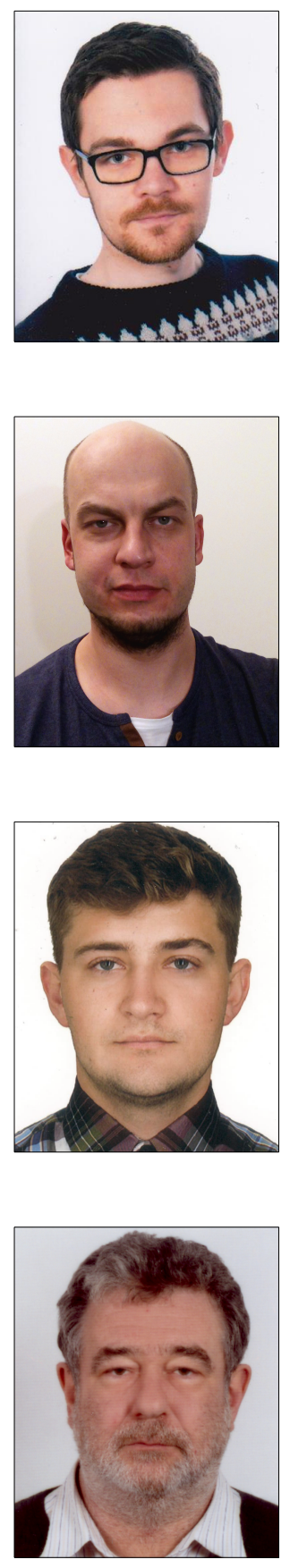

Valeriy Pleechystyy was born in 1994 in Semenivka, Ukraine. In 2017, he received his M.Sc. degree in Physics from the Ivan Franko National University of Lviv. Currently he is a PhD Student at Gdańsk University of Technology, Gdańsk, Poland. Research interests: the mechanism and kinetics of phase formation in composites at the liquid-crystal interface.

Jaroslaw Rybicki is Professor in Theoretical and Computational Physics in the Faculty of Applied Physics and Mathematics at Gdańsk University of Technology, Gdańsk, Poland. His field of interest covers the structure of disordered systems (oxide glasses and liquid metals and alloys), phase transitions (condensation from gas phase, premelting phenomena), and the mechanical properties of nanostructures (mechanisms of plastic deformation, formation and motion of dislocations, molecular mechanisms of friction). Main research tools: classical and quantum-classical simulations with particles, stochastic geometry methods. 